\title{
A single rack cloud centre with unprecedented power and thermal efficiency
}

\section{Chih-hsun Lin ${ }^{1}$}

Institute of Physics, Academia Sinica

128, Sec.2, Academia Rd, Taipei 115, Taiwan

E-mail: chihhsun. Iin@phys.sinica.edu.tw

\section{George Chu}

ASGC, Acedemia Sinica

128, Sec.2, Academia Rd, Taipei 115, Taiwan

E-mail: george.chu@tegrid.org

\section{Pei-rong Tsai}

Institute of Physics, Academia Sinica

128, Sec.2, Academia Rd, Taipei 115, Taiwan

E-mail: sky315134@hotmail.com

\section{Chan-hin long}

ASGC, Acedemia Sinica

128, Sec.2, Academia Rd, Taipei 115, Taiwan

E-mail:chiong@me.com

\section{Felix Lee}

ASGC, Acedemia Sinica

128, Sec.2, Academia Rd, Taipei 115, Taiwan

E-mail:felix@twgrid.org

\section{Eric Yen}

ASGC, Acedemia Sinica

128, Sec.2, Academia Rd, Taipei 115, Taiwan

E-mail:eric.yen@tegrid.org

\section{Shih-chang Lee}

Institute of Physics, Academia Sinica

128, Sec.2, Academia Rd, Taipei 115, Taiwan

E-mail:phsclee@phys.sinica.edu.tw

\section{${ }^{1}$ Speaker}


In this paper, we report the development of fanless single rack data centre (SRDC) which is noisefree and of efficiency in power, energy and operation. SRDC could be deployed as a building block according to different needs and close to users to reduce the latency. Distributed cloud infrastructure constituted by these SRDCs could provide federated computing capability as a large cloud centre. Private cloud could be also implemented according to the application needs.

The first prototype of fanless SRDC with a dedicated cooling system is accomplished and verified. It consists of fanless computing nodes with dual CPUs, fanless 12 bays disk servers and a fanless $10 \mathrm{G}$ switch with fibre interfaces. Test results show that heat generated by components in each server can bring out effectively by the conductional cooling. The temperature of CPUs can be kept within $60^{\circ} \mathrm{C}$ under the full load condition.

The cooling system modified from the existing air conditioner can achieved PUE $<1.25$ in the summer condition.

International Symposium on Grids and Clouds 2017 -ISGC 2017-

5-10 March 2017

Academia Sinica, Taipei, Taiwan 


\section{Introduction}

We developed the fanless single rack data center (SRDC) which is noise-free and of efficiency in power, energy and operation. By combining with the distributed cloud operating system (DiCOS) [1], SRDC could be deployed as a building block according to different needs and close to the users to reduce the latency. Distributed cloud infrastructure constituted by these SRDCs could provide federated computing capability as a large cloud center. Private cloud could be also implemented according to the application needs. Today, a standard rack with SRDCs could have more than 1,000 CPU cores and 1 Petabyte disk space with PUE less than 1.2. Together with DiCOS, the fanless SRDC achieves the synergy of a distributed infrastructure with scalability, flexibility and minimized latency.

\section{Design concept}

The development goal is the high efficiency of power usage in a SRDC. We accomplished this goal in two different ways. First, the power waste shall be minimized. A SRDC often uses an uninterrupted power supply (UPS) to prevent service interrupts due to electricity glitches. Two additional AC-DC and DC-AC conversions are needed in a UPS since battery is a DC device. It causes at least $10-20 \%$ power loss before delivering power to equipment in a SRDC. To solve this issue and keep the same reliability, we introduce a lithium battery pack into the 12VDC power line of IT equipment. The second way to achieve the high power usage efficiency is to minimize the power consumption of cooling system. In order to improve the efficiency of cooling system, the conductional cooling concept is introduced as shown in Figure 1. Two rack sidewalls are used
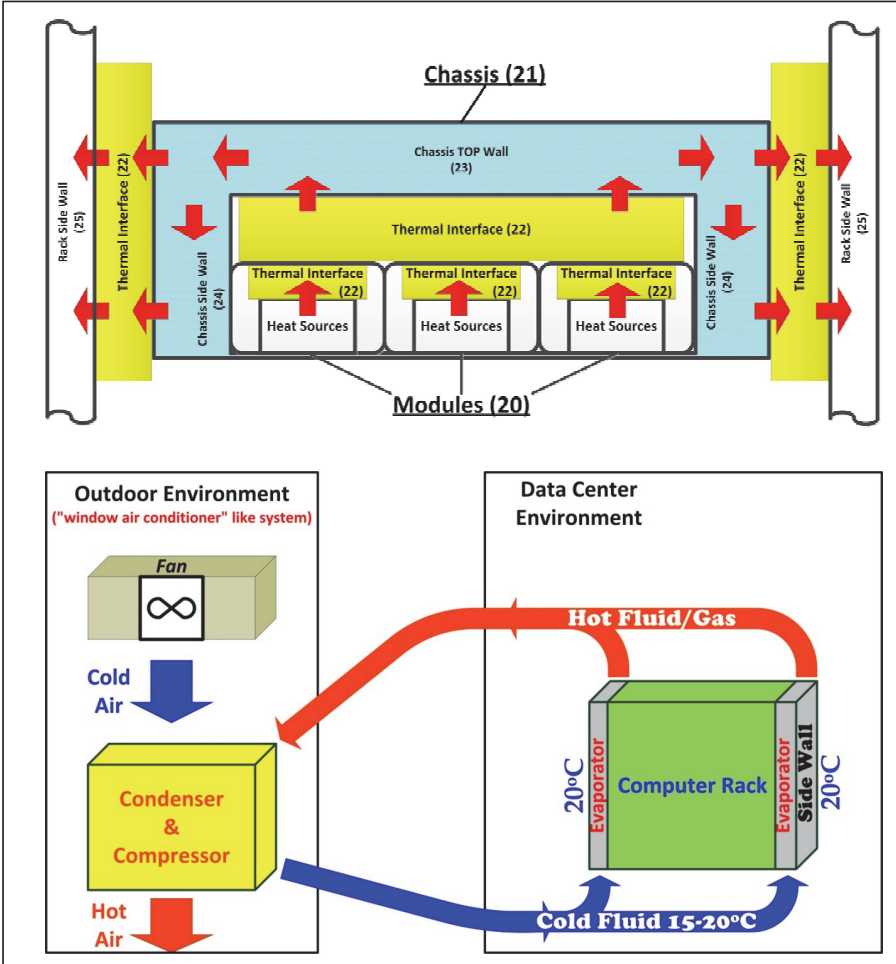

Figure 1: Conductive cooling architecture for a single rack data center. The top figure shows how the component heat is transferred to two sidewalls. The bottom figure how the heat is moved to environment. Heat flow is indicated by arrows. as the evaporator. Heat generated in IT equipment is brought to sidewalls and then is took away by the coolant flow. The outdoor unit with a condenser and a compressor is just like an outdoor unit of commercial air conditioner. In such way, it is not only to improve the cooling system efficiency but also reduces the power consumption of IT equipment.

\section{Implementation}

\subsection{Fanless rack}

As shown in Figure 1, we proposed to use two side walls of a rack as evaporators in the cooling loop. This evaporator was made by an $\mathrm{Al}$ alloy plate with copper pipes in it for the coolant loop. Its size is $770 \mathrm{~mm} \times 1020 \mathrm{~mm}$, 
which can be fit into a standard 19" $25 \mathrm{U}$ rack. In order to have a uniform temperature distribution, there are four coolant loops in an evaporator. Each cooling loop consists 4 to 5 pairs of copper pipes.

\subsection{Cooling system for fanless SRDC}

It is modified from an outdoor unit of a commercial air conditioner. However, the system design and control logics of this cooling system for fanless SRDC are quite different to the commercial air conditioner. The evaporation temperature of commercial air conditioner is usually well below $10-15^{\circ} \mathrm{C}$. In this cooling system, the

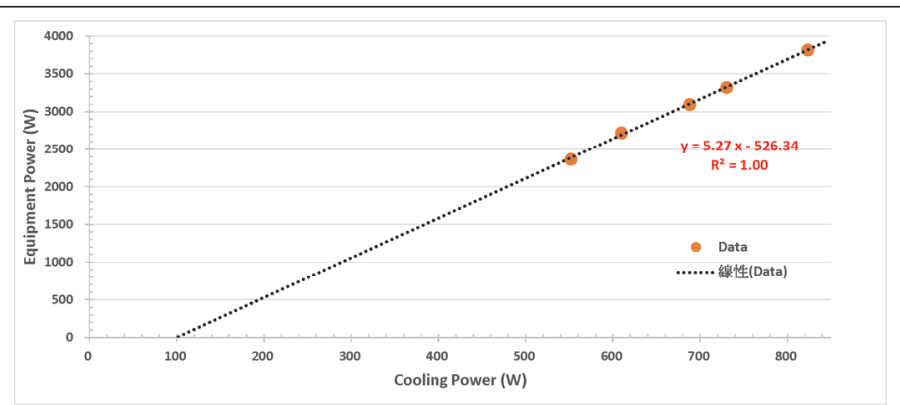

Figure 2: Efficiency of cooling system for fanless SRDC. It was tested up to $3.8 \mathrm{~kW}$. The PUE reaches 1.22 .

evaporation temperature is set to be around $25^{\circ} \mathrm{C}$. This is a significant improvement to the compressor efficiency. Therefore, we chose a small Tatung compressor with $2 \mathrm{~kW}$ capability in the specification but use it up to $4 \mathrm{~kW}$ of heat load. In addition, the expansion valve was installed close to each rack sidewall (evaporator). In such way, it reduces the loss of cooling capability due to a long pipe between the outdoor unit and the evaporator. It also improve the equalization of coolant flow between two evaporators.

In order to verify the efficiency of this cooling system precisely, heaters were installed on two evaporators to emulate the heat load. It was tested from $2.4 \mathrm{~kW}$ to $3.8 \mathrm{~kW}$ and the environmental temperature is around $32^{\circ} \mathrm{C}$ to $35^{\circ} \mathrm{C}$. Test results are shown in Figure 2. It shows the good linear relation between the cooling system power and the equipment power. Their ratio ranges from 4.29 to 4.64 , which presents PUE from 1.215 to 1.233 .

\subsection{Fanless server}

The fanless server is modified from SuperMicro 1U server. Its specifications are listed below:

- Dual Intel Xeon E5-2630L CPU with 6 cores.

- Sixteen (eight) 8GB (16GB) DDR3 1333 ECC DIMM

- Dual onboard 10G SFP+ Ethernet interfaces and RAID controller.

- Two hard disks with mirror configuration.

The original cooling design is based on six cooling fans. It takes $50 \mathrm{~W}$ to $72 \mathrm{~W}$, which is a significant fraction of overall power (150W-300W). Hot components are identified by the thermal camera. The conductional cooling methods for different components are summarized below:

- For high power consumption components like CPU and RAID/Ethernet controllers, the heat pipe is used to remove heat from them to chassis sidewalls.

- The Al alloy heat sink is used to conduct heat from memory DIMMs to the chassis top panel.

- A $3 \mathrm{~mm}$ thermal pad is put under the server mother board to conduct heats from rest of components to the chassis bottom plate. 
- For hard disks, power supplies and BBP modules, the Al alloy heat sink is used to conduct heat from them to chassis sidewalls. In order to keep the possibility to remove them without chassis opening, a special fin type heat sink joint is designed.

- The same fin structure is used to conduct heat from chassis sidewalls
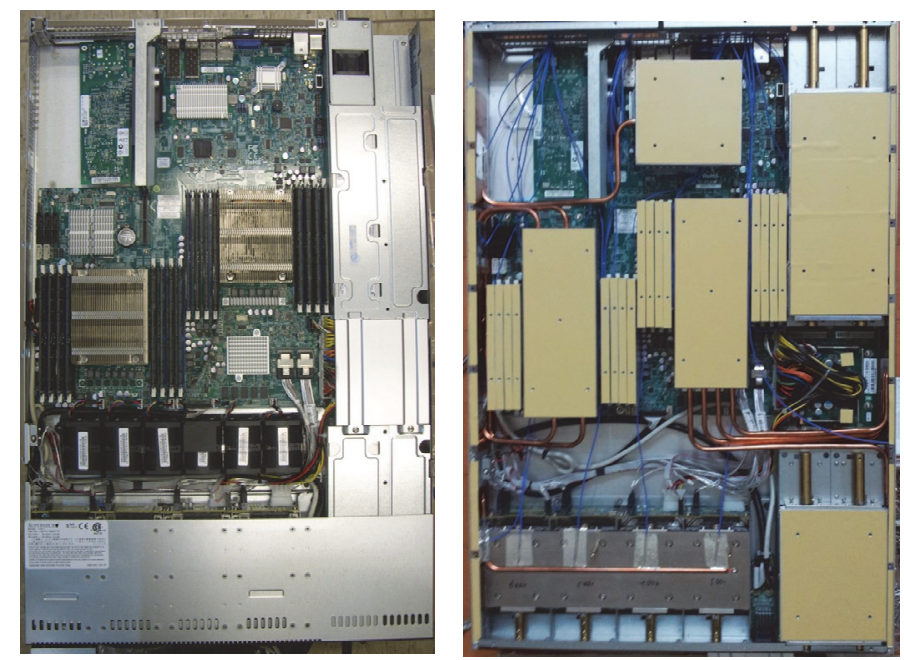

Figure 3: (left) Original cooling design with 6 fans installed. (right) New conductional cooling chassis. All fans are removed. to rack sidewalls.

Therefore, the fanless server can be inserted and removed from the fanless rack easily.

Figure 3 shows this SuperMicro server before and after the modification for conductional cooling.

\subsection{Fanless disk server}

The specification of SuperMicro 12bay disk servers is:

- The CPU is Intel Atom processor C2750 with 8 cores.

- Four 16GB DDR3 1333MHz SDRAM DIMMs with ECC.

- One add-on card with 10GB SFP+ Ethernet interface.

- Twelve 3.5" hot-swap 4TB hard disks.

The same cooling methods are applied to produce fanless disk servers. Figure 4 shows the fanless disk server.

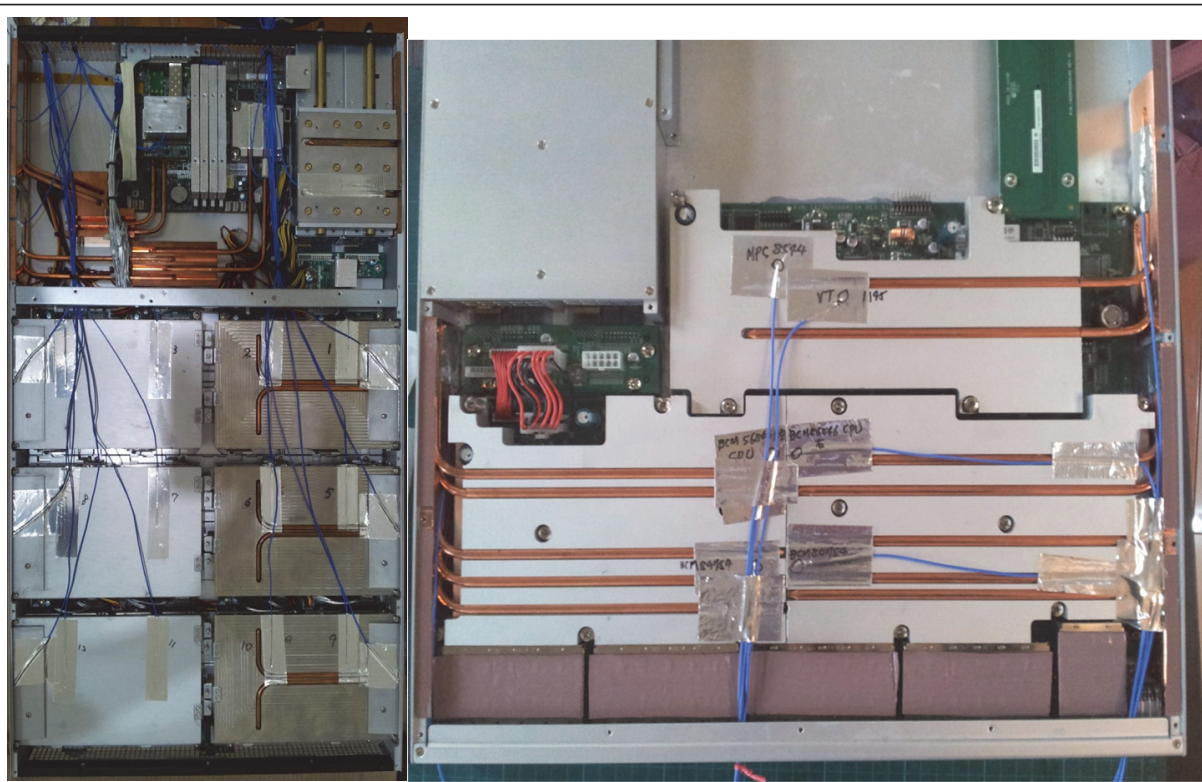

Figure 4: Fanless disk server with 12 3.5" 4TB hard disks and fanless switch with 48 10Gb SFP+ ports. 


\subsection{Fanless disk switch}

Fanless switch is modified from a SuperMicro switch with 48 10Gb (SFP+) ports, 4 48Gb (QSFP) ports and $21 \mathrm{~Gb}$ ports with RJ45 connectors. It provides all necessary functions for network switching, security and management. Figure 4 shows the fanless switch.

1. Tests of fanless rack with fanless IT equipment installed

The 25U fanless rack with 9 fanless servers, 3 fanless disk servers with 36 hard disks in total, one fanless switch with $4810 \mathrm{~Gb}$ $\mathrm{SFP}+$ ports and one fanless $1 \mathrm{~Gb}$ switch with RJ45 ports for management is shown in Figure 5. It has been tested with the cooling system under the fully loaded condition. All 9 fanless servers with

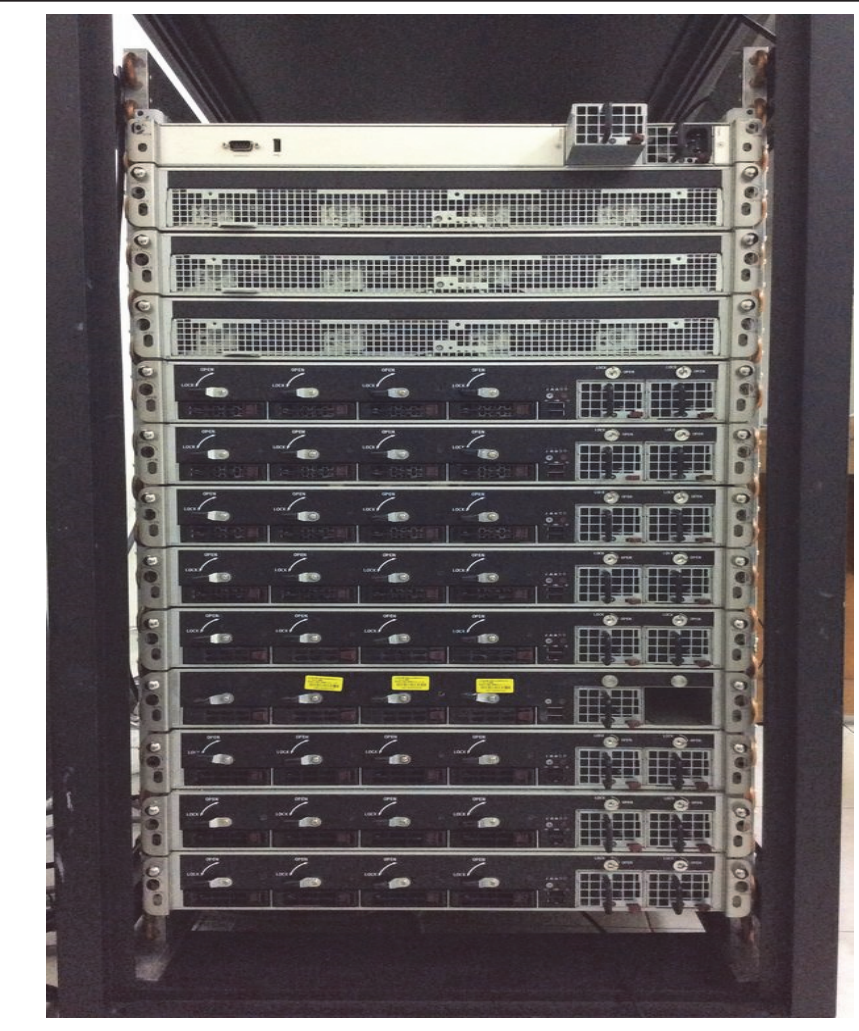

Figure 5: Fanless rack with all fanless equipment installed. the hyper thread option enabled are loaded with the SPEC CPU 2006 benchmark program. The temperature of rack sidewall was kept to around $23^{\circ} \mathrm{C}$. The environmental temperature is around $30^{\circ} \mathrm{C}$. The peak power of all equipment is around $2600 \mathrm{~kW}$. All equipment ran well in this test and was kept in the good thermal condition. Figure 6 and 7 shows the temperature of CPU and DDR3 DIMM as examples. The CPU temperatures were kept between $50^{\circ} \mathrm{C}$ to $55^{\circ} \mathrm{C}$ for $\mathrm{CPU} 2$ and $55^{\circ} \mathrm{C}$ to $60^{\circ} \mathrm{C}$ for $\mathrm{CPU} 1$. The reason

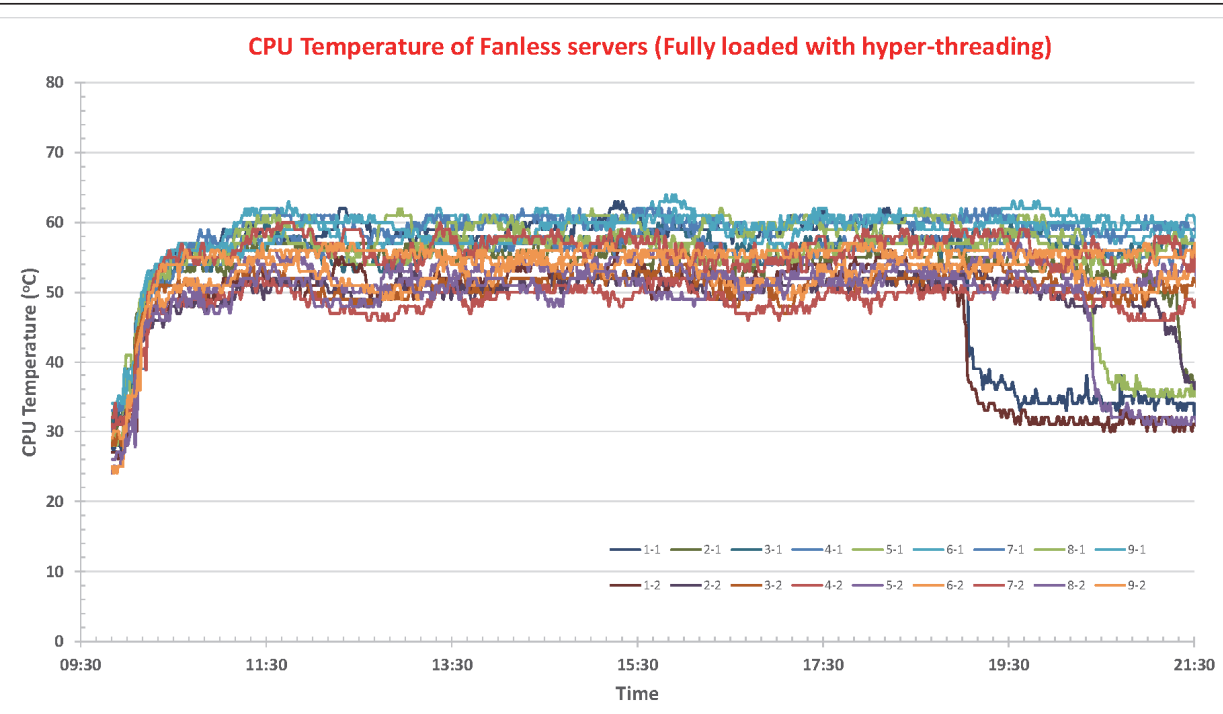

Figure 6: Temperature of CPUs during the load test. All CPU temperatures are kept between $50^{\circ} \mathrm{C}$ to $60^{\circ} \mathrm{C}$, which is well below the limit $\left(70^{\circ} \mathrm{C}\right)$. 


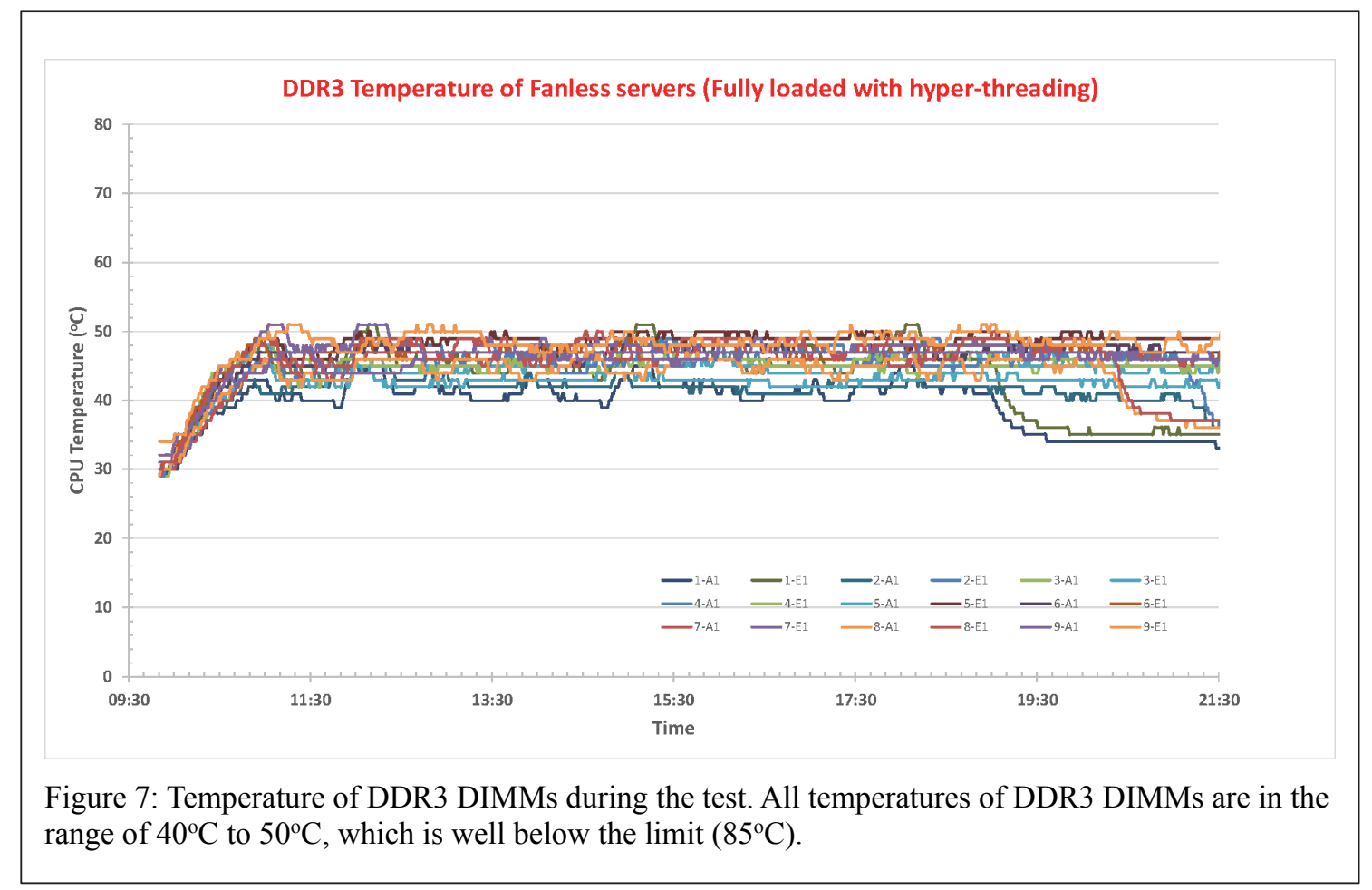

is that CPU2 is closed to the chassis sidewall. CPU1 is located in the middle of a fanless server. It needs a longer heat pipe. The temperature of DDR3 DIMM is below $50^{\circ} \mathrm{C}$. Therefore, the thermal performance can be further improved if the whole system is designed with the conditional cooling concept.

\section{Summary}

The first prototype of fully installed fanless SRDC with a dedicated cooling system is accomplished and verified. The PUE $<1.25$ in the summer condition is achieved. Test results show that heat generated by components in each server can bring out effectively by the conductional cooling. The temperature of CPUs can be kept within $60^{\circ} \mathrm{C}$ under the full load condition.

\section{References}

[1] Eric Yen et al., Distributed Cloud Operating System (DiCOS) Development at Academia Sinica, in proceedings of ISGC conference PoS (ISGC 2016) 038. 